\title{
Evaluation of real-world persistence of propiverine and trospium chloride in treatment of overactive bladder in Germany
}

This article was published in the following Dove Medical Press journal: Research and Reports in Urology

\section{Sandra Murgas' \\ Daniela Adolf ${ }^{2}$}

'APOGEPHA Arzneimittel GmbH, Dresden, Germany; ${ }^{2}$ StatConsult

Gesellschaft für klinische und Versorgungsforschung $\mathrm{mbH}$, Magdeburg, Germany
Correspondence: Sandra Murgas APOGEPHA Arzneimittel $\mathrm{GmbH}$, Kyffhäuserstraße 27, 01309 Dresden, Germany

Tel +4935I 3363484

Fax +49 35I 3363440

Email smurgas@apogepha.de

\begin{abstract}
Treatment persistence poses a crucial criterion for therapeutic success. Like in many other chronic diseases, in overactive bladder (OAB) syndrome also, many patients discontinue their treatment for diverse reasons. In order to evaluate the persistence in medication, this paper presents data of propiverine extended release (ER) and trospium chloride immediate release (IR) for three consecutive quarters, thus giving an insight into the probability of treatment discontinuation for these two drugs. Prescription data from German health insurances were analyzed. The frequency of follow-up prescriptions and drop-outs for propiverine ER and trospium chloride IR were compared for treatment-naïve, restarted, and switched patients after treatment initiation (quarter 1) for a period of 9 months (three consecutive calendar quarters). In all analyzed quarters, the percentage of follow-up prescriptions was significantly higher for propiverine ER than for trospium chloride IR. The chance of a follow-up prescription was also significantly higher for propiverine ER than for trospium chloride IR, although, in general, both drugs showed a decrease of follow-up prescriptions over time. This comparison of propiverine ER (once-daily administration) and trospium chloride IR (in a multiple dose administration) in patients with $\mathrm{OAB}$ syndrome demonstrates that there are substantial differences in the odds of a follow-up prescription. The longer a patient adheres to a therapeutic treatment plan, the better the chances for improvement of symptoms and having a positive impact on activities of daily living. Keywords: overactive bladder, propiverine, trospium chloride, treatment continuation, adherence
\end{abstract}

\section{Introduction}

The overactive bladder (OAB) syndrome is defined as urinary urgency, usually joined by increased daytime frequency and nocturia, with or without urgency urinary incontinence, in absentia of urinary tract infection or other obvious pathology. ${ }^{1}$

Because of its chronic nature, OAB syndrome requires sufficient long-term treatment, and antimuscarinic drugs are presently the first choice. ${ }^{2}$ Generally, patients' adherence and persistence to long-term medication plans is considered substandard, but especially for OAB, it is considerably low. ${ }^{3}$ The reason for this phenomenon is multifactorial. Since OAB can have such a negative impact on activities of daily living, therapy expectation is typically high, even though the outcome in chronic disease might be improvement rather than extinction of symptoms. Any side effects of applied medication might also play a role in discontinuation in some cases. ${ }^{4}$ Discontinuation of treatment often results in symptom relapse or retreatment.

One of the presently prescribed drugs for OAB treatment is propiverine extended release (ER). The active ingredient, propiverine hydrochloride, blocks 
muscarinic receptors. In addition, it inhibits calcium influx and modulates intracellular calcium in the bladder smooth muscle cells, which then leads to musculotropic spasmolysis. ${ }^{5}$ In contrast, trospium chloride blocks the muscarinic receptors only. Trospium chloride is the most frequently prescribed drug for this indication in Germany. Even though the mechanisms slightly differ, both drugs have a similar side effect profile.

This article presents data evaluating the probability of follow-up prescriptions of propiverine ER and trospium chloride immediate release (IR) in three consecutive calendar quarters in Germany.

\section{Materials and methods}

Prescription data from German health insurances were analyzed. The frequency of follow-up prescriptions and drop-outs for propiverine ER and trospium chloride IR were compared in a cross table. Significant differences are presented according to chi-squared tests. The differences are quantified by ORs and the corresponding 95\% CIs. These values represent the chance of a follow-up prescription of propiverine ER vs one of trospium chloride IR. The analyses were performed quarterly for the entire patient group as well as for three subgroups:

- Treatment naïve: patients with new prescriptions, no treatment for these symptoms before.

- Restarts: patients with prescriptions in the past, but without prescription of the same drug in the last 6 months.

- Switches: patients switching from another drug of this class (the following antimuscarinic drugs were included: darifenacin, fesoterodine, oxybutynin, solifenacin, tolterodine IR, tolterodine ER, trospium ER) to the analyzed drug.

All analyses were performed with the software SAS 9.2 (SAS Institute Inc., Cary, NY, USA) and intentionally calculated to a full significance level of 5\%. For this type of analysis, ethical approval and informed consent from patients are not required.

\section{Results}

The prescription data were evaluated for four quarters in 2013 (Q1-Q4). The data were assessed according to "yes" (there was a follow-up prescription) and "no" (there was none within 90 days). A total of 54,385 patients' data were evaluated for Q1. Of these, 6,706 patients were treated with propiverine ER, of which 2,615 were treatment naïve, 2,158 were restarts, and 1,935 were switches. Also, 47,679 patients had a prescription of trospium chloride IR; in this group of patients were 27,196 new prescriptions, 15,227 restarts, and 5,256 patients who switched from another medication.

In all analyzed quarters (Q2-Q4), the percentage of follow-up prescriptions was significantly higher for propiverine ER than for trospium chloride IR, even though the difference decreased from one quarter to the next. In Q2, the OR for a follow-up prescription for propiverine ER vs trospium chloride IR was $3.73(3.48,4.00)$, leading to the conclusion that out of 100 patients treated with propiverine ER, 77 would get a follow-up prescription. For trospium chloride IR, there would only be 48 out of 100 patients. In Q3, the odds of a follow-up prescription for propiverine ER were $90 \%$ higher than for trospium chloride IR (OR $=1.90$ $[1.80,2.00])$. Meaning, out of 100 patients, 36 would get a follow-up prescription for propiverine ER, and 23 out of 100 patients would get one for trospium chloride IR. Even though the contrast was less explicit in Q4, there was still a 60\% higher chance for a follow-up prescription for propiverine ER (28 patients of 100) compared to trospium chloride IR (20 patients of 100), OR $=1.60(1.51,1.69)$.

The consolidated results of the subgroup analysis are presented in Table 1. The three subgroups, treatment naïve, restarts, and switches, were also analyzed for follow-up prescription in the quarters Q2-Q4 and the ORs were evaluated.

As shown in Table 1, tendencies were the same as for the entire patient group. Although both drugs showed a decrease of prescription over time, the chance of a followup prescription for propiverine ER was significantly higher than for trospium chloride IR. Only in case of the switches after Q2, the differences were not statistically detectable anymore. The most prominent effects were apparent for the patients in the restart group. In Q2 in this group, the OR was $4.05(3.53,4.65)$, meaning that 83 patients out of 100 would get a follow-up prescription for propiverine ER and only 55 out of 100 patients would get it for trospium chloride IR.

In Figure 1A, B, the ORs are presented with the corresponding $95 \% \mathrm{CI}$ for the analyzed three quarters. The reference line at point 1.0 shows the point where the chance of a follow-up prescription for both medications would be equal. All described outcomes are visible, for example, the high ORs in Q2 and the decreased differences over the time of the analysis from Q2 to Q4. Also, in the last quarter in the subgroup "switches", the CI of the OR includes the reference value of 1.0 (Figure 1B). 
Table I Follow-up prescriptions (Q2-Q4) for propiverine ER and trospium chloride IR for all patients and patients in the subgroups

\begin{tabular}{|c|c|c|c|c|c|c|c|c|}
\hline \multirow{2}{*}{$\begin{array}{l}\text { Patient } \\
\text { group }\end{array}$} & \multirow[t]{2}{*}{ Quarter } & \multirow{2}{*}{$\begin{array}{l}\text { Follow-up } \\
\text { prescription }\end{array}$} & \multicolumn{2}{|c|}{ Propiverine ER } & \multicolumn{2}{|c|}{ Trospium chloride IR } & \multirow[t]{2}{*}{$P$-value } & \multirow[t]{2}{*}{ OR $(95 \% \mathrm{Cl})$} \\
\hline & & & $\mathbf{n}$ & $\%$ & $\mathbf{n}$ & $\%$ & & \\
\hline \multirow[t]{6}{*}{ All } & \multirow[t]{2}{*}{ Q2 } & Yes & 5,673 & 84.60 & 28,391 & 59.55 & $<0.001$ & $3.73(3.48,4)$ \\
\hline & & No & 1,033 & 15.40 & 19,288 & 40.45 & & \\
\hline & \multirow[t]{2}{*}{ Q3 } & Yes & 2,845 & 42.42 & 13,327 & 27.95 & $<0.001$ & $1.9(1.8,2)$ \\
\hline & & No & $3,86 I$ & 57.58 & 34,352 & 72.05 & & \\
\hline & \multirow[t]{2}{*}{ Q4 } & Yes & 2,154 & 32.12 & 10,907 & 22.88 & $<0.001$ & $\mathrm{I} .6(\mathrm{I} .5 \mathrm{I}, \mathrm{I} .69)$ \\
\hline & & No & 4,552 & 67.88 & 36,772 & 77.12 & & \\
\hline \multirow{6}{*}{$\begin{array}{l}\text { Treatment } \\
\text { naïve }\end{array}$} & \multirow[t]{2}{*}{ Q2 } & Yes & 2,074 & 79.31 & 14,436 & 53.08 & $<0.001$ & $3.39(3.07,3.74)$ \\
\hline & & No & 541 & 20.69 & 12,760 & 46.92 & & \\
\hline & \multirow[t]{2}{*}{ Q3 } & Yes & 873 & 33.38 & 6,118 & 22.50 & $<0.001$ & $1.73(1.58 .1 .88)$ \\
\hline & & No & $\mathrm{I}, 742$ & 66.62 & 21,078 & 77.50 & & \\
\hline & \multirow[t]{2}{*}{ Q4 } & Yes & 654 & 25.01 & 4,894 & 18.00 & $<0.001$ & $1.52(1.38,1.67)$ \\
\hline & & No & $1,96 \mid$ & 74.99 & 22,302 & 82.00 & & \\
\hline \multirow[t]{6}{*}{ Restarts } & \multirow[t]{2}{*}{ Q2 } & Yes & $1,9 \mid 4$ & 88.78 & 10,068 & 66.12 & $<0.001$ & $4.05(3.53,4.65)$ \\
\hline & & No & 242 & 11.22 & 5,159 & 33.88 & & \\
\hline & \multirow[t]{2}{*}{ Q3 } & Yes & 970 & 44.99 & 4,752 & 31.21 & $<0.001$ & I.8 (I.65, I.98) \\
\hline & & No & 1,186 & 55.01 & 21,078 & 68.79 & & \\
\hline & \multirow[t]{2}{*}{ Q4 } & Yes & 719 & 33.35 & 3,957 & 25.99 & $<0.001$ & $1.43(1.29,1.57)$ \\
\hline & & No & $\mathrm{I}, 437$ & 66.65 & $\mathrm{II}, 270$ & 74.01 & & \\
\hline \multirow[t]{6}{*}{ Switches } & \multirow[t]{2}{*}{ Q2 } & Yes & 1,685 & 87.08 & 3,887 & 73.95 & $<.001$ & $2.37(2.05,2.75)$ \\
\hline & & No & 250 & 12.92 & 1,369 & 26.05 & & \\
\hline & \multirow[t]{2}{*}{ Q3 } & Yes & 1,002 & 51.78 & 2,457 & 46.75 & 0.002 & $1.22(\mathrm{I} . \mathrm{I}, \mathrm{I} .36)$ \\
\hline & & No & 933 & 48.22 & 2,799 & 53.25 & & \\
\hline & \multirow[t]{2}{*}{ Q4 } & Yes & 781 & 40.36 & 2,056 & 39.12 & 0.338 & $1.05(0.95,1.17)$ \\
\hline & & No & $\mathrm{I}, 154$ & 59.64 & 3,200 & 60.88 & & \\
\hline
\end{tabular}

Abbreviations: ER, extended release; IR, immediate release.

\section{Discussion}

Treatment of a chronic disease and, therefore, a long-term medication plan represents a challenge in terms of patients' adherence and persistence. This analysis confirmed what others have also shown: adherence to $\mathrm{OAB}$ medication generally decreases over time. ${ }^{6}$ It can be speculated that the high discontinuation rate may be attributable to a combination of patient expectations and the relation of efficacy and drug tolerability. Therefore, differences between drugs are conceivable. This analysis demonstrated that $>80 \%$ of patients taking propiverine ER have had follow-up prescription in Q2, compared to $<60 \%$ of the patients who were treated with trospium chloride IR. The percentage decreased with the next quarters, but the difference between propiverine ER and trospium chloride IR was significant over the entire time of analysis. When splitting up into subgroups, the tendencies stayed the same. The differences lessened over the quarters, but only in the group of "switches", the difference between the drugs was lower, and thus not significant in Q4. The reasons for the changing numbers of prescriptions were not analyzed. It might be reasonably assumed that treatment with propiverine ER was either more efficient or was associated with less side effects or both. A previous study demonstrated that propiverine had better odds in a continued treatment in urinary incontinence. ${ }^{7}$ In a German database analysis of 26,000 patients with urinary incontinence, the following drugs were identified as being prescribed: darifenacin, fesoterodine, oxybutynin, propiverine, solifenacin, tolterodine, and trospium chloride. The authors analyzed the probability of treatment discontinuation and compared all antimuscarinics with oxybutynin as the reference. Only propiverine had a significantly lower HR than oxybutynin itself. ${ }^{7}$ This means that the chance of discontinuation is lower, which is in line to what has been described here, that the continuation of therapy is higher. These findings could be interpreted as support for the data shown here.

There are some limitations of this study. The discontinuation of the medication, and thus, the lack of follow-up prescriptions cannot be reasoned in detail, as the motives of discontinuation were not reported and thus not analyzed. Also, the patient groups were not differentiated in detail, such as age classes, sex, or comorbidities. 
A

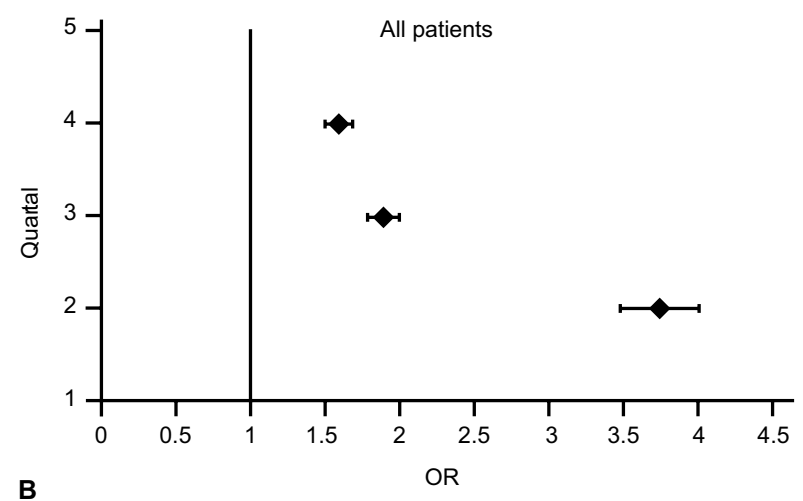

B
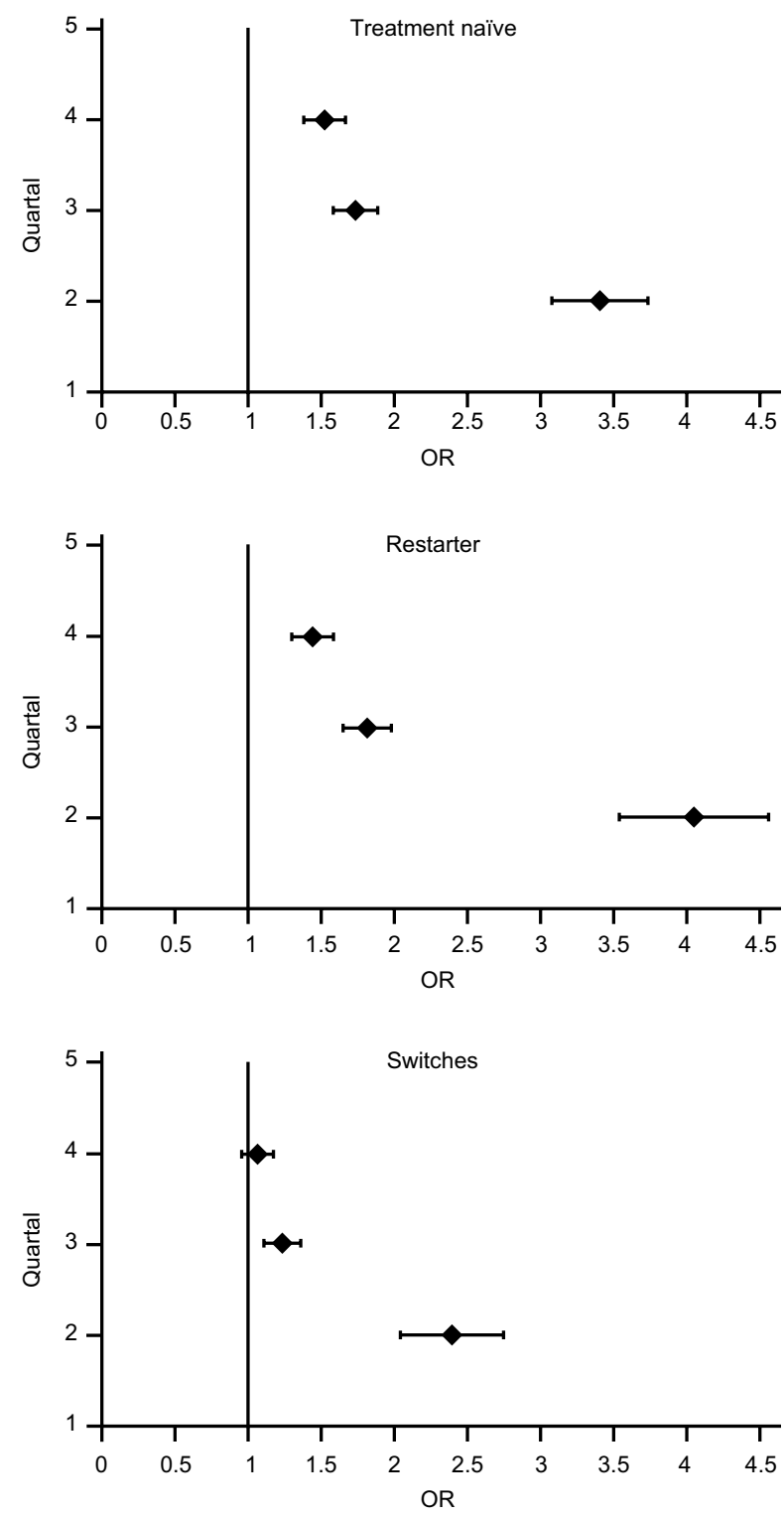

Figure I ORs of follow-up prescription for propiverine ER vs trospium chloride IR. Notes: ORs of follow-up prescription for propiverine ER vs trospium chloride IR for (A) the entire patient group from quarter 2 to 4 and (B) the subgroups "Treatment naïve", "Restarter", and "Switches". Reference line at 1.0 indicates equal odds for follow-up prescriptions for both medications. Bars indicate $95 \% \mathrm{Cls}$.

Abbreviations: ER, extended release; IR, immediate release.

\section{Conclusion}

Several medications are available for treating OAB syndrome. Unfortunately, long-term medication plans often lead to discontinuations by the patients, diminishing the therapeutic success of the treatment. By comparing the two drugs propiverine ER (once-daily administration) and trospium chloride IR (in a multiple dose administration), this analysis shows that there are significant differences in the odds of a follow-up prescription. In all evaluated groups within this analysis, entire patient group or the subgroups, the chance of a follow-up prescription for propiverine ER was higher than for trospium chloride IR. Individual reasons for discontinuation might be diverse. The longer a patient adheres to a treatment plan, the better the chance for improvement of symptoms and having a positive impact on activities of daily living.

\section{Acknowledgment}

The authors would like to thank Dr Beate Walter for assistance with manuscript preparation.

\section{Disclosure}

The authors report no conflicts of interest in this work.

\section{References}

1. Abrams P, Artibani W, Cardozo L, et al. Reviewing the ICS 2002 terminology report: the ongoing debate. Neurourol Urodyn. 2009;28(4):287.

2. Veenboer PW, Bosch JL. Long-term adherence to antimuscarinic therapy in everyday practice: a systematic review. J Urol. 2014;191(4):1003-1008.

3. Yeaw J, Benner JS, Walt JG, Sian S, Smith DB. Comparing adherence and persistence across 6 chronic medication classes. J Manag Care Pharm. 2009;15(9):728-740.

4. Kim TH, Choo MS, Kim YJ, Koh H, Lee KS. Drug persistence and compliance affect patient-reported outcomes in overactive bladder syndrome. Qual Life Res. 2016;25(8):2021-2029.

5. McKeage K. Propiverine: a review of its use in the treatment of adults and children with overactive bladder associated with idiopathic or neurogenic detrusor overactivity, and in men with lower urinary tract symptoms. Clin Drug Investig. 2013;33(1):71-91.

6. Basra RK, Wagg A, Chapple C, et al. A review of adherence to drug therapy in patients with overactive bladder. BJU Int. 2008;102(7):774-779.

7. Kalder M, Pantazis K, Dinas K, Albert US, Heilmaier C, Kostev K. Discontinuation of treatment using anticholinergic medications in patients with urinary incontinence. Obstet Gynecol. 2014;124(4):794-800. 
Research and Reports in Urology

\section{Publish your work in this journal}

Research and Reports in Urology is an international, peer-reviewed, open access journal publishing original research, reports, editorials, reviews and commentaries on all aspects of adult and pediatric urology in the clinic and laboratory including the following topics: Pathology, pathophysiology of urological disease; Investigation and treatment of urological disease; Pharmacology of drugs used for the treatment of urological disease. The manuscript management system is completely online and includes a very quick and fair peer-review system, which is all easy to use. Visit http://www.dovepress.com/testimonials.php to read real quotes from published authors.

Submit your manuscript here: https://www.dovepress.com/research-and-reports-in-urology-journal 